\title{
Phenotypic and molecular characterisation of CDK13-related congenital heart defects, dysmorphic facial features and intellectual developmental disorders
}

Bret L. Bostwick ${ }^{1 *}$, Scott McLean ${ }^{1,13}$, Jennifer E. Posey ${ }^{1}$, Haley E. Streff ${ }^{1}$, Karen W. Gripp ${ }^{2}$, Alyssa Blesson², Nina Powell-Hamilton², Jessica Tusi ${ }^{2}$, David A. Stevenson ${ }^{3}$, Ellyn Farrelly ${ }^{3}$, Louanne Hudgins ${ }^{3}$, Yaping Yang 1,4, Fan Xia ${ }^{1,4}$, Xia Wang ${ }^{1,4}$, Pengfei Liu ${ }^{1,4}$, Magdalena Walkiewicz ${ }^{1,4}$, Marianne McGuire ${ }^{1}$, Dorothy K. Grange ${ }^{5}$, Marisa V. Andrews ${ }^{5}$, Marybeth Hummel ${ }^{6}$, Suneeta Madan-Khetarpal ${ }^{7}$, Elena Infante ${ }^{7}$, Zeynep Coban-Akdemir ${ }^{1}$, Karol Miszalski-Jamka ${ }^{8}$, John L. Jefferies ${ }^{9}$, Members of the Undiagnosed Diseases Network ${ }^{10}$, Jill A. Rosenfeld ${ }^{1}$, Lisa Emrick, Kimberly M. Nugent ${ }^{1,13}$, James R. Lupski 1,11,12,14, John W. Belmont ${ }^{1}$, Brendan Lee ${ }^{1}$ and Seema R. Lalani ${ }^{1}$

\begin{abstract}
Background: De novo missense variants in CDK13 have been described as the cause of syndromic congenital heart defects in seven individuals ascertained from a large congenital cardiovascular malformations cohort. We aimed to further define the phenotypic and molecular spectrum of this newly described disorder.

Methods: To minimise ascertainment bias, we recruited nine additional individuals with CDK13 pathogenic variants from clinical and research exome laboratory sequencing cohorts. Each individual underwent dysmorphology exam and comprehensive medical history review.

Results: We demonstrate greater than expected phenotypic heterogeneity, including 33\% (3/9) of individuals without structural heart disease on echocardiogram. There was a high penetrance for a unique constellation of facial dysmorphism and global developmental delay, as well as less frequently seen renal and sacral anomalies. Two individuals had novel CDK13 variants (p.Asn842Asp, p.Lys734Glu), while the remaining seven unrelated individuals had a recurrent, previously published p.Asn842Ser variant. Summary of all variants published to date demonstrates apparent restriction of pathogenic variants to the protein kinase domain with clustering in the ATP and magnesium binding sites.

Conclusions: Here we provide detailed phenotypic and molecular characterisation of individuals with pathogenic variants in CDK13 and propose management guidelines based upon the estimated prevalence of anomalies identified.
\end{abstract}

Keywords: CDK13, CHDFIDD, De novo variant, Neurodevelopmental disorders, Agenesis of the corpus callosum, Hypertelorism, Developmental delay, Cyclin-dependent kinase, Undiagnosed Diseases Network

\footnotetext{
* Correspondence: bostwick@bcm.edu

${ }^{1}$ Department of Molecular and Human Genetics, Baylor College of Medicine,

6701 Fannin St, Suite 1560, Houston, TX 77030, USA

Full list of author information is available at the end of the article
} 


\section{Background}

Congenital heart defects, facial dysmorphism and intellectual developmental disorder (CHDFIDD) is a newly described syndrome caused by de novo variants in CDK13 [1]. The syndrome was discovered as part of a large exome sequencing cohort of 1891 individuals with congenital heart defects. All seven initially reported children had structural congenital heart disease with either atrial and/or ventricular septal defects or pulmonary valve abnormalities. Individuals were reported to have a recognisable facial gestalt characterised by a small mouth, thin upper lip, posteriorly rotated ears, epicanthal folds, upslanted palpebral fissures and hypertelorism. All individuals had global developmental delay and/or intellectual disability. Brain imaging abnormalities included agenesis of the corpus callosum and aplasia of the cerebellar vermis. A subsequent report identified additional individuals with de novo variants in CDK13 that reached genomewide significance in a second large exome sequencing cohort of 4293 families containing individuals with developmental disorders [2]. However, dysmorphology and organ-specific detailed phenotypic information was not available.

CDK13 encodes a 1512 amino acid protein kinase involved in the regulation of gene expression by controlling the phosphorylation status and activity of splicing regulators [3, 4]. It is part of a family of 20 different ATP-dependent serine-threonine protein kinases regulating cell-cycle progression and gene expression [5]. $C D K 12$ and $C D K 13$ are thought to have evolved by duplication of a common gene ancestor; however, both kinases appear to operate in separate complexes in mammalian cells $[6,7]$. In developing mouse embryos, Cdk12 and Cdk13 regulate axonal elongation [8], suggesting an important role in neuronal development. All pathogenic variants in CDK13 to date are clustered in the highly conserved serine-threonine kinase domain with molecular modelling predicting perturbation of either ATP binding, magnesium binding or interaction with cyclin $\mathrm{K}[1]$.

Although CDK13-related CHDFIDD was initially thought to be an extremely rare disorder, 20 patients have been identified in less than one year from initial disease gene discovery $[1,2]$. Here, we aim to provide the first detailed phenotypic summary of CDK13-related CHDFIDD ascertained through clinical and research exome sequencing pipelines.

\section{Methods}

\section{Participants}

This study was approved by the Baylor College of Medicine Institutional Review Board for Human Subjects Research. The initial individual was diagnosed by exome sequencing after enrolment in the Undiagnosed Diseases Network at the Baylor College of Medicine Clinical Site. In order to provide comprehensive phenotypic and molecular characterisation, additional individuals with $C D K 13$ pathogenic variants were recruited from clinical exome sequencing laboratory cohorts and one individual was ascertained through review of the Baylor-Hopkins Center for Mendelian Genomics (BHCMG) exome variant database. We received informed consents to proceed with publication for nine unrelated individuals. Eight of the nine individuals underwent dysmorphology exam by an American Board of Medical Genetics and Genomics board-certified physician. Comprehensive medical records, including physical exam findings, laboratories, imaging studies, developmental status and disease natural history were reviewed. When possible, original laboratory reports or imaging studies were obtained for review.

\section{DNA preparation and sequence analysis}

In all nine individuals, $C D K 13$ variants were initially detected by exome sequencing. In eight individuals, the remaining extracted DNA was used to perform Sanger sequencing confirmation. In one individual (Individual 1009), no additional extracted DNA was available, but the exome data were manually reviewed and considered to be reliable for inclusion (24 variant reads out of 53 total reads). For Sanger sequencing, venous blood samples were collected for DNA extraction from peripheral blood leukocytes (PBL). Total genomic DNA was extracted with the Puregene DNA extraction kit (Gentra Systems, Inc. Minneapolis, MN, USA) according to the manufacturer's protocol. Genomic DNA from each individual was used for polymerase chain reaction (PCR) amplification with primer design dependent upon variant genomic location. PCR amplification was performed for 35 cycles from $50 \mathrm{ng}$ of total genomic DNA extracted from PBL. All PCR products were purified. Direct sequence analysis of PCR products was performed in both the forward and reverse directions using automated fluorescence dideoxy sequencing methods. Sequencing profiles were inspected visually to detect heterozygous changes. The base numbering refers to the A of the ATG start codon as position 1 and the nomenclature is based on the convention recommended by the Human Genome Variation Society. A variant was determined to be de novo if it was not present in either maternal or paternal samples.

\section{Results}

\section{Genotype}

Nine individuals were found to have missense substitutions in highly conserved amino acid positions of CDK13. Eight of the nine patients were confirmed to have de novo variants. Parental samples were unavailable for Individual 1009, who had a previously described 
pathogenic variant (c.2525A > G). No genotype-phenotype correlations were found between variant location and severity or spectrum of disease manifestations; however, the number of individuals enrolled in this study limited the power of this analysis.

Seven of nine patients shared the same variant (c.2525A > G, p.Asn842Ser), previously reported as pathogenic $[1,2]$. Two missense changes (c.2524A $>\mathrm{G}$, p.Asn842Asp and c.2200A > G, p.Lys734Glu) are novel de novo variants. Each of these variants perturbs an amino acid residue previously reported in a pathogenic variant $[1,2]$, but results in different amino acid substitutions. None of the variants were found in ExAC or gnomAD databases (accessed on 17 June 2017). Nucleotide variant, predicted protein alteration, zygosity and variant history are summarised in Table 1 . Those variants previously reported are accompanied by PMID reference in Table 1. No additional pathogenic, likely pathogenic or de novo variants were reported in any of the clinical laboratory exome sequencing reports (Additional File 1: Table S1).

Including this report, there are 29 individuals with CDK13 pathogenic variants $[1,2]$ and this report], with 27 proven de novo and two of unknown inheritance, secondary to unavailable parental samples. All variants are predicted to impact the protein kinase domain (amino acids 697-1029), with clustering in the ATP- binding and magnesium-binding sites as demonstrated in Fig. 1. More than half of the variants $(15 / 29)$ perturb the wild-type asparagine residue at amino acid position 842, suggesting its importance in magnesium interaction. The absence of loss-of-function variants combined with the clustering of missense variants within a single protein domain in affected individuals suggests a possible gain-of-function disease mechanism.

\section{Phenotype}

Phenotypic characterisation was performed by medical history, imaging, laboratory and dysmorphology review. Medical records were reviewed comprehensively with the goal of better understanding the clinical spectrum of CDK13-related CHDFIDD. Facial examination for potential dysmorphology was performed for all patients with available photographs and is summarised in Table 2 . Several highly penetrant facial features were identified in the majority of patients, suggesting a potentially recognisable pattern or 'facial gestalt'. A few of the patients $(\mathrm{n}=3)$ had been previously suspected to have Kabuki syndrome, demonstrating the similarity to this condition. The distinctive facial features of CDK13-related CHDFIDD include hypertelorism, epicanthal folds, highly arched eyebrows, wide nasal bridge, short columella, thin upper lip and abnormal ears. All patients had at least three key features and $92 \%$ had four or more of these seven key

Table 1 Genotype results. All individuals (1001-1009) were found to have CDK13 variants initially by either research or clinical exome sequencing

\begin{tabular}{|c|c|c|c|c|c|c|}
\hline Sex, ID & Age (years) & Nucleotide variant & Protein alteration & De novo & Zygosity & Variant history (PMID) \\
\hline$F, 1001$ & 2 & c. $2524 A>G$ & p.N842D & Yes & Het & Novel variant \\
\hline$F, 1002$ & 8 & c. $2525 A>G$ & p.N842S & Yes & Het & 27479907 \\
\hline M, 1003 & 4 & c. $2525 \mathrm{~A}>\mathrm{G}$ & p.N842S & Yes & Het & 27479907 \\
\hline M, 1004 & 2 & c. $2525 \mathrm{~A}>\mathrm{G}$ & p.N842S & Yes & Het & 27479907 \\
\hline$F, 1005$ & 14 & c. $2525 \mathrm{~A}>\mathrm{G}$ & p.N842S & Yes & Het & 27479907 \\
\hline$F, 1006$ & 2 & c. $2525 A>G$ & p.N842S & Yes & Het & 27479907 \\
\hline M, 1007 & 17 & c. $2525 A>G$ & p.N842S & Yes & Het & 27479907 \\
\hline M, 1008 & 0.5 & c. $2200 A>G$ & p.K734E & Yes & Het & Novel variant \\
\hline M, 1009 & 38 & c. $2525 A>G$ & p.N842S & Unknown & Het & 27479907 \\
\hline$F, 265645^{a}$ & 7 & c. $2525 A>G$ & p.N842S & Yes & Het & 27479907 \\
\hline$F, 265813^{a}$ & 0.2 & c. $2525 \mathrm{~A}>\mathrm{G}$ & p.N842S & Yes & Het & 27479907 \\
\hline$F, 259460^{a}$ & 3 & c. $2525 \mathrm{~A}>\mathrm{G}$ & p.N842S & Yes & Het & 27479907 \\
\hline$M, 262889^{a}$ & 8 & $c .2149 \mathrm{G}>\mathrm{A}$ & p.G717R & Yes & Het & 27479907 \\
\hline$F, 271894^{a}$ & 5 & $c .2140 G>C$ & p.G714R & Yes & Het & 27479907 \\
\hline$F, 258830^{a}$ & 12 & c. $2252 \mathrm{G}>\mathrm{A}$ & p.R751Q & Yes & Het & 27479907 \\
\hline$M, 270818^{a}$ & 1 & c. $2525 A>G$ & p.N842S & Yes & Het & 27479907 \\
\hline
\end{tabular}

Where possible, all variants were confirmed by Sanger sequencing. Note that a paternal sample was not available for ID 1009, thus de novo status is unknown. Age refers to age at last assessment 


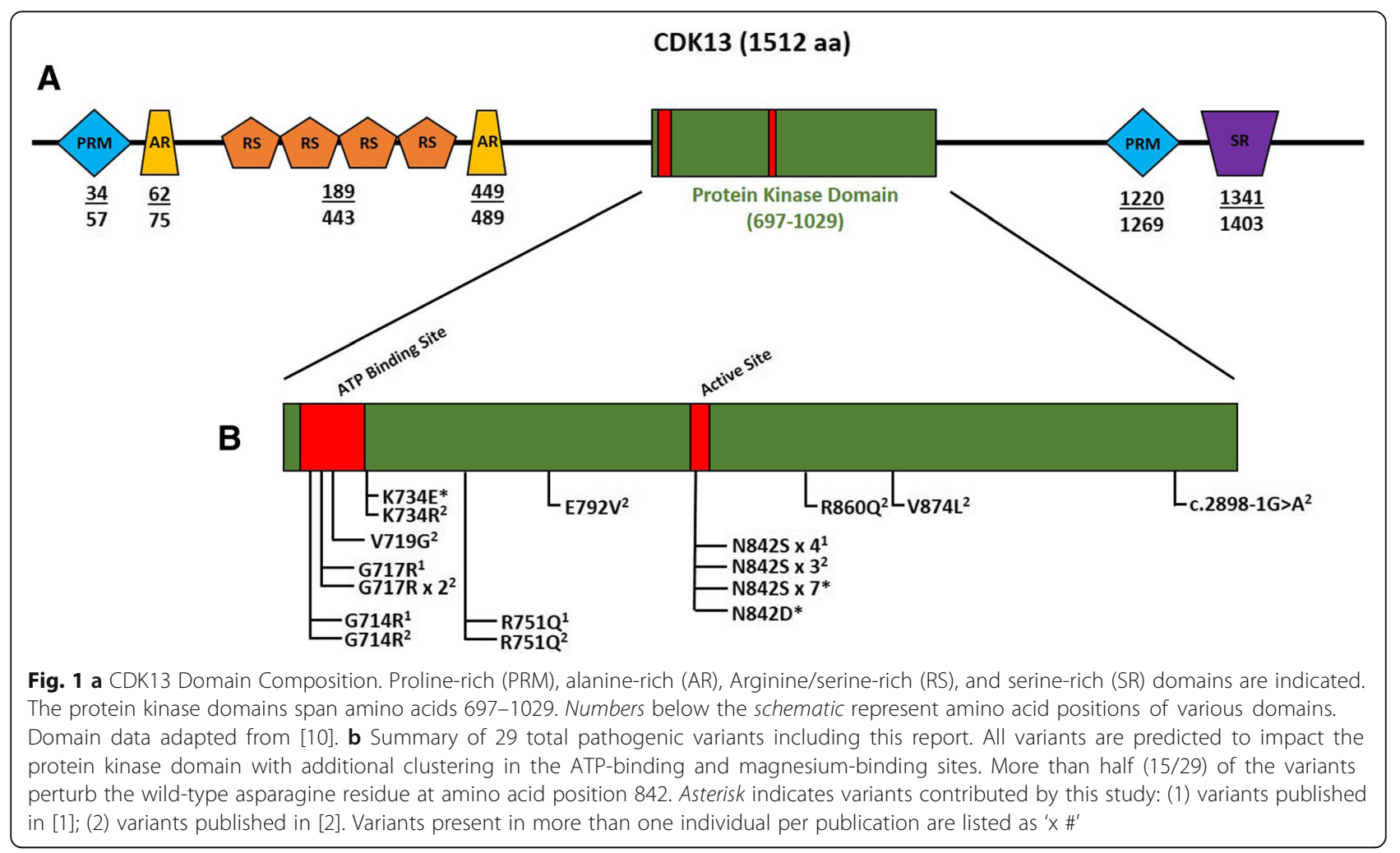

features, suggesting that this condition typically presents with a consistent and potentially recognisable constellation of dysmorphic features, as illustrated in Fig. 2. In addition, ear abnormalities were universally present and included low-set positioning, posterior angulation, overfolded helices and various simplified morphologies with cupping (Fig. 3a).

Strabismus (69\%), abnormal tone including hypotonia (69\%) or spasticity (15\%), and musculoskeletal abnormalities (62\%) were commonly observed (Table 2). Although sacral anomalies were present on examination in a minority of patients $(n=4)$, the unusual nature of the manifestations may help in syndrome recognition. Sacral abnormalities ranged from simple dimples or sacral clefting, to spina bifida, to a striking bony sacral prominence with apical slit as seen in Fig. 3b.

Table 3 summarises the cardiac, brain, renal and skeletal abnormalities and provides details regarding growth and development. All patients had gross motor and language delays and were diagnosed with either developmental delay or intellectual disability. The degree of intellectual impairment ranged from mild to severe. Several had poor weight gain or short stature. Four patients had microcephaly. Among those who had brain MRI, central nervous system abnormalities were almost universal (10/11) and included periventricular gliosis $(n=3)$, dysgenesis of the corpus callosum $(n=4)$, spinal cord syrinx $(n=2)$, cerebellar tonsillar abnormalities $(n=2)$ and diminished white matter volume $(n=1)$. Renal abnormalities seen in three patients included duplicated collecting systems, dilated collecting systems and fused renal ectopia. Spinal abnormalities were observed in $31 \%(5 / 16)$ of individuals, ranging from scoliosis or hyperlordosis $(n=3)$ to one individual who had multiple cervical spinal fusions and a large haemangioma of the L1 vertebral body. Loose or hypermobile joints were reported in the majority of individuals; however, due to a lack of objective criteria between examiners, these findings were not aggregated in tabular form.

Since CDK13-related CHDFIDD was initially discovered in a large exome sequencing congenital heart disease cohort [1], all initial patients were selected for study based upon their expressed cardiac disease. In our series of nine patients, congenital cardiac disease was present in two-thirds (6/9) of the individuals confirming that it is a prominent, but not invariant, component of the syndrome. In the remaining three individuals (1003, 1005, 1007; ages 4, 14 and 17 years, respectively), echocardiograms did not reveal evidence for structural cardiac disease. In aggregate, cardiac involvement was present in $81 \%$ of individuals (13/16). Defects seen in multiple patients include atrial septal defects (8/16), ventricular septal defects (4/16), pulmonary valve abnormalities $(2 / 16)$ and hypoplastic left pulmonary artery (2/16). One patient had Ebstein's anomaly with an abnormal tricuspid valve. It is important to note that the oldest individual in this report (Individual 1009) is 


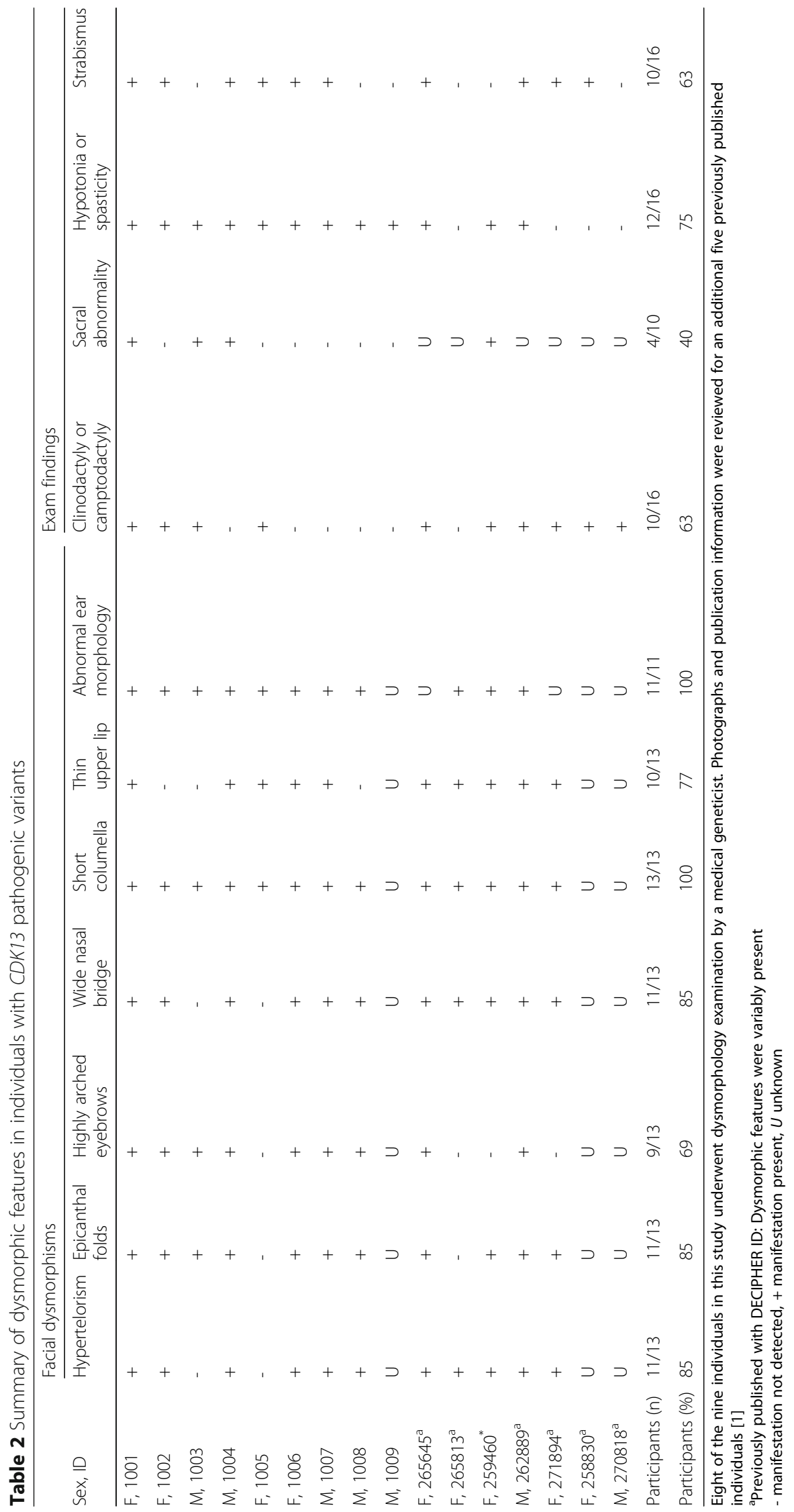




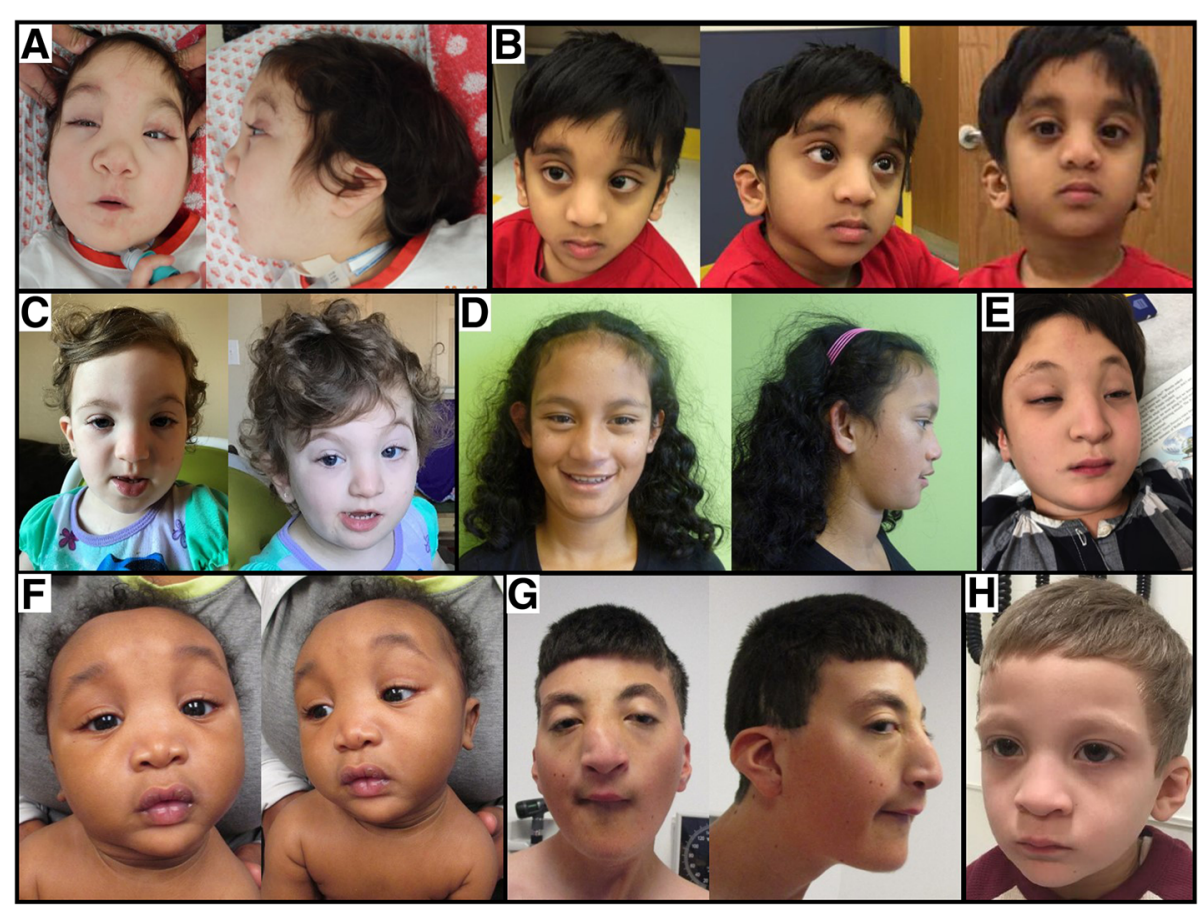

Fig. 2 Craniofacial and dysmorphology features in individuals with pathogenic CDK13 variants. Patients share a facial gestalt which in some cases include hypertelorism, epicanthal folds, highly arched eyebrows, widened nasal bridge, short columella, thin upper lip and dysplastic ears. a Study ID 1001. b Study ID 1004. c Study ID 1006. d Study ID 1005. e Study ID 1002. f Study ID 1008. g Study ID 1007. h Study ID 1003

38 years old and has left ventricular non-compaction and sick sinus syndrome requiring pacemaker implantation, after having initially presented as a child with bicuspid aortic valve, aortic stenosis and aortic insufficiency. The cardiomyopathy and electrical disturbance in the oldest individual may suggest age-related penetrance of additional cardiac sequelae; however, phenotypic extrapolation should be cautioned as these sequelae were only seen in a single individual. Alternatively, the detection of leftventricular non-compaction in adulthood may be due to improvements in imaging technology over time.
Additional low-frequency findings in our cohort that are not included in tabular form include hypothyroidism $(\mathrm{n}=1)$, small chest circumference $(\mathrm{n}=2)$, malignant hyperthermia $(\mathrm{n}=1)$, craniosynostosis $(\mathrm{n}=2)$ and club foot deformity $(\mathrm{n}=2)$.

\section{Discussion}

After the recent discovery [1] of CDK13 as the cause of congenital heart defects, facial dysmorphism and intellectual developmental disorder (CHDFIDD), we aimed to further delineate the phenotypic spectrum, dysmorphology

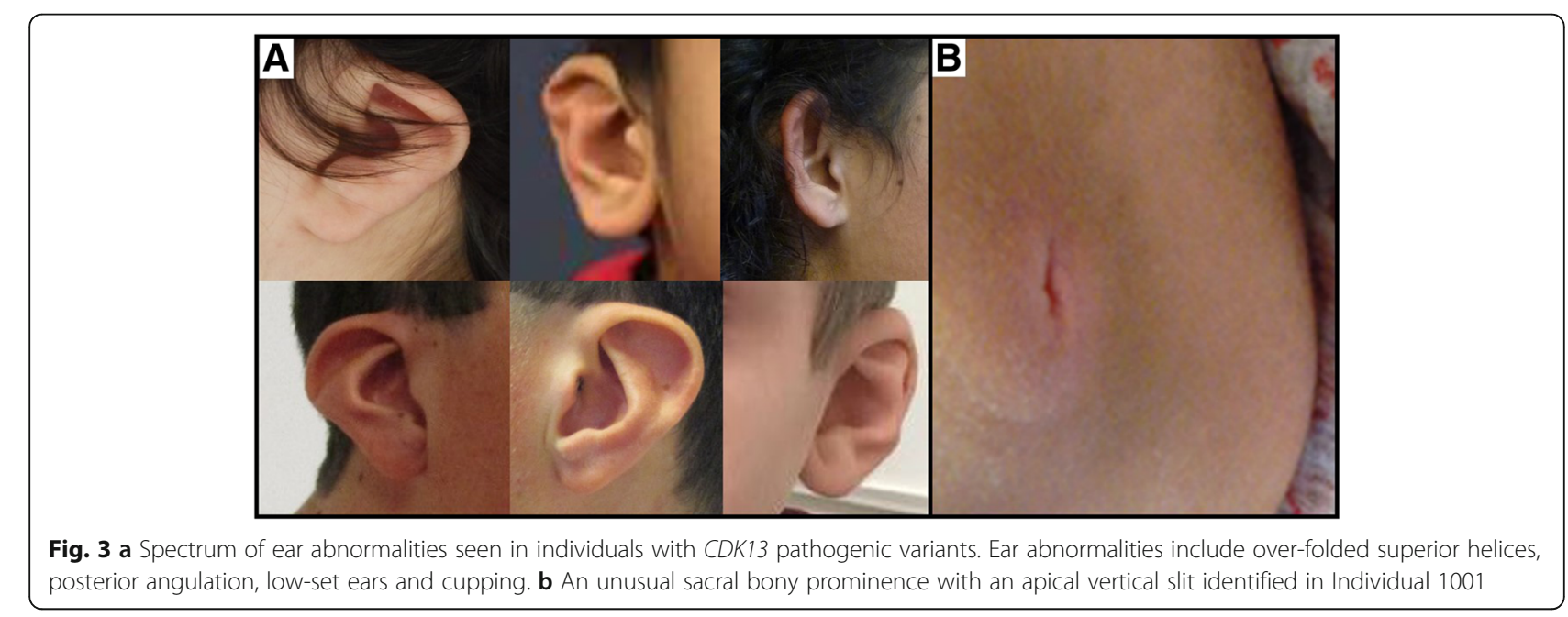




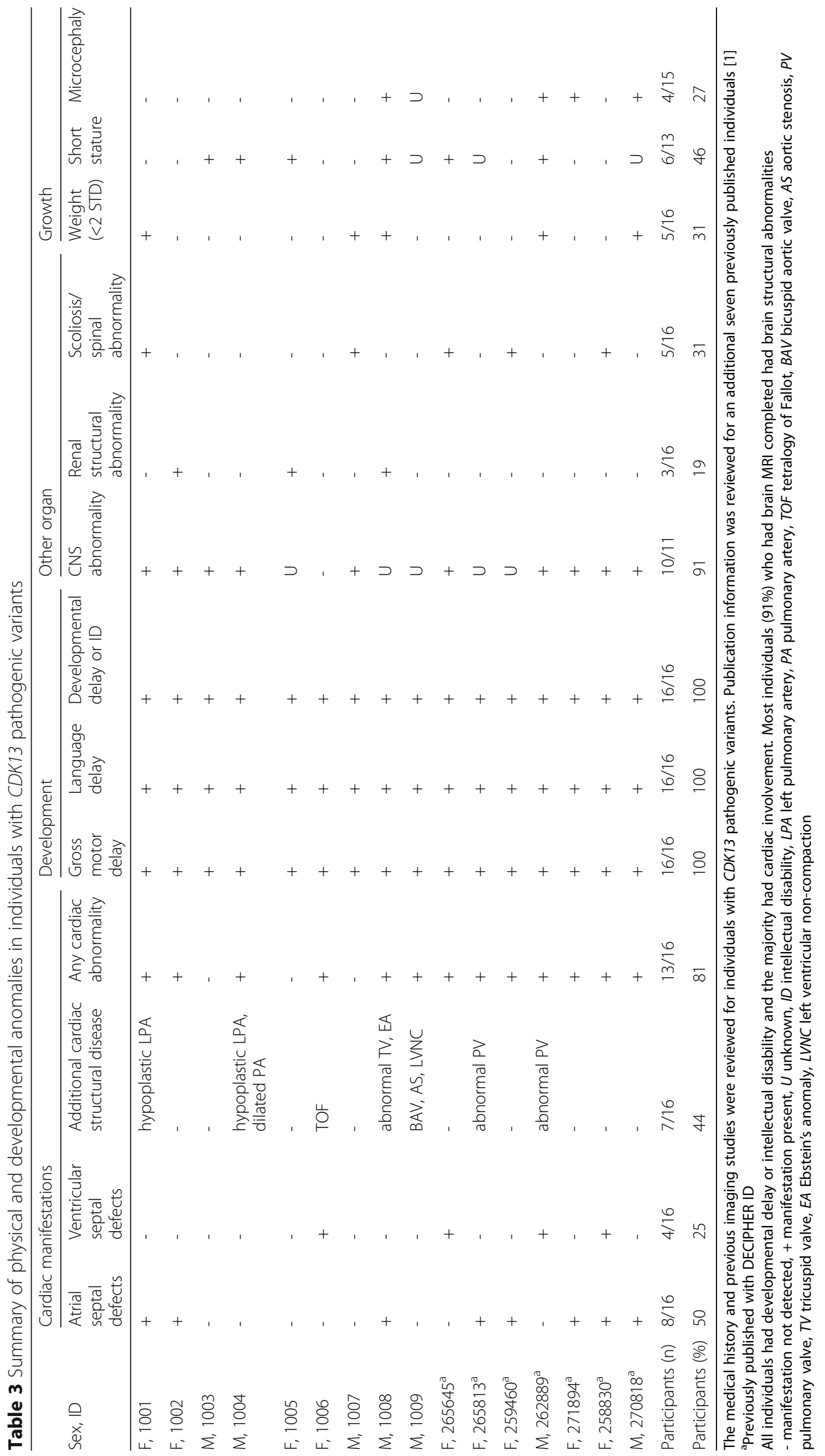


and medical co-morbidities of this newly described syndrome. In order to minimise ascertainment bias, we took a genotype-first approach and recruited nine additional unpublished individuals with CDK13 pathogenic variants detected by clinical or research exome sequencing. Our results confirm that congenital heart disease, neurodevelopmental disorders and facial dysmorphisms are important components. Emerging from this detailed phenotyping, we emphasise the potential clinical significance of other organ involvement including renal abnormalities, spinal and sacral anomalies, and widen the spectrum for neurological structural abnormalities and cardiac abnormalities. We suspect that the recurrent craniofacial dysmorphisms may reliably constitute a recognisable facial gestalt. Interestingly, some individuals in the series had previously been considered by initial clinical examination to have features consistent with Kabuki syndrome. Given the clinical feature overlap, CDK13 genotyping should be considered in individuals clinically suspected to have Kabuki syndrome but who lack molecular confirmation. The compilation of these clinical features and congenital anomalies affords an opportunity to propose initial management suggestions based on the calculated prevalence of multi-system involvement.

\section{Proposed initial management upon diagnosis of CDK13- related CHDFIDD}

Given the prevalence of cardiac, neurological, renal, ocular and skeletal abnormalities in individuals with $C D K 13$ pathogenic variants, consideration of echocardiogram, brain MRI, renal ultrasound and ophthalmology evaluation are warranted in all patients after initial molecular diagnosis. Although this study is limited by our phenotypic sample size $(n=16)$, there is ample evidence to recommend this minimum initial medical evaluation. Additionally, any sacral abnormalities identified on physical exam should prompt additional evaluation including sacral imaging as indicated. Given that developmental delay was present in all individuals, therapies should be initiated at the youngest possible age to maximise developmental potential. Ascertainment of additional individuals and understanding the natural history of this syndrome will further refine these proposed management guidelines.

\section{Increasing need for reverse clinical genomics}

Historically, most disease gene discovery occurred after the gathering of a large cohort of individuals with a remarkably similar phenotype. It was common for a syndrome to be identified, named and well-delineated prior to gene discovery. With the advent of next-generation sequencing, many new gene discoveries are originating from large disease-specific sequencing cohorts often containing thousands of individuals. In these new studies, syndromes are identified first molecularly instead of phenotypically. This experimental design has created a new demand for 'reverse clinical genomics', where disease-gene directed cohorts are then studied phenotypically to delineate the syndromic associations and appropriate medical management. Recent papers [9] have employed a similar approach, demonstrating the need for subsequent detailed clinical evaluation as a prerequisite to understanding the complete phenotypic spectrum.

\section{Conclusions}

Extensive phenotyping using a genotype-first approach and aggregated clinical phenotyping information from participants with pathogenic CDK13 variants enables syndrome delineation and initial construction of clinical management guidelines. We highlight the recognisable facial characteristics, congenital and neurodevelopmental anomalies and propose initial management guidelines for this Mendelian disorder. The exome sequencing rare variant data underscore the importance of diseaseassociated pathogenic variant clustering around the ATP-binding and magnesium-binding sites and emphasises that all reported pathogenic variants to date are missense substitutions in the protein kinase domain.

\section{Additional file}

Additional file 1: Table S1. All pathogenic, likely pathogenic or de novo variants identified on the clinical genetics laboratory report for Individuals 1001-1008. (PDF $450 \mathrm{~kb})$

\section{Acknowledgements}

Sincere appreciation is extended to the patients and families described herein for their willingness to participate in this research. We are also grateful to the support of the many collaborators we reached out to in order to ascertain this cohort. No additional compensation was received for these contributions.

Members of the Undiagnosed Diseases Network (UDN) include Mercedes E. Alejandro, Carlos A. Bacino, Ashok Balasubramanyam, Lindsay C. Burrage, Gary D. Clark, William J. Craigen, Shweta U. Dhar, Lisa T. Emrick, Brett H. Graham, Neil A. Hanchard, Mahim Jain, Seema R. Lalani, Brendan H. Lee, Richard A. Lewis, Mashid S. Azamian, Paolo M. Moretti, Sarah K. Nicholas, Jordan S. Orange, Jennifer E. Posey, Lorraine Potocki, Jill A. Rosenfeld, Daryl A. Scott, Alyssa A. Tran, Jing Zhang, Tiphanie P. Vogel, Bret L. Bostwick, Shan Chen, Susan L. Samson, Hugo J. Bellen, Michael F. Wangler, Shinya Yamamoto, Christine M. Eng, Donna M. Muzny, Patricia A. Ward, Yaping Yang, David B. Goldstein, Nicholas Stong, Yong-hui Jiang, Allyn McConkieRosell, Loren DM. Pena, Kelly Schoch, Vandana Shashi, Rebecca C. Spillmann, Jennifer A. Sullivan, Nicole M. Walley, Alan H. Beggs, Lauren C. Briere, Cynthia M. Cooper, Laurel A. Donnell-Fink, Elizabeth L. Krieg, Joel B. Krier, Sharyn A. Lincoln, Joseph Loscalzo, Richard L. Maas, Calum A. MacRae, J. Carl Pallais, Lance H. Rodan, Edwin K. Silverman, Joan M. Stoler, David A. Sweetser, Chris A. Walsh, Cecilia Esteves, Ingrid A. Holm, Isaac S. Kohane, Paul Mazur, Alexa T. McCray, Matthew Might, Rachel B. Ramoni, Kimberly Splinter, David P. Bick, Camille L. Birch, Braden E. Boone, Donna M. Brown, Daniel C. Dorset, Lori H. Handley, Howard J. Jacob, Angela L. Jones, Jozef Lazar, Shawn E. Levy, J. Scott Newberry, Molly C. Schroeder, Kimberly A. Strong, Elizabeth A. Worthey, Jyoti G. Dayal, David J. Eckstein, Sarah E. Gould, Ellen M. Howerton, Donna M. Krasnewich, Laura A. Mamounas, Teri A. Manolio, John J. Mulvihill, Anastasia L. Wise, Tiina K. Urv, Ariane G. Soldatos, Matthew Brush, Jean-Philippe F. Gourdine, Melissa Haendel, David M. Koeller, Jennifer E. Kyle, Thomas O. Metz, Katrina M. Waters, Bobbie-Jo M. Webb-Robertson, Euan A. Ashley, Jonathan A. Bernstein, Annika M. Dries, Paul G. Fisher, Jennefer N. Kohler, Daryl M. Waggott, Matthew T. Wheeler, 
Patricia A. Zornio, Patrick Allard, Hayk Barseghyan, Esteban C. Dell'Angelica, Katrina M. Dipple, Naghmeh Dorrani, Matthew R. Herzog, Hane Lee, Stan F. Nelson, Christina GS. Palmer, Jeanette C. Papp, Janet S. Sinsheimer, Eric VilainChristopher J. Adams, Elizabeth A. Burke, Katherine R. Chao, Mariska Davids, David D. Draper, Tyra Estwick, Trevor S. Frisby, Kate Frost, Valerie Gartner, Rena A. Godfrey, Mitchell Goheen, Gretchen A. Golas, Mary G. Gordon, Catherine A. Groden, Mary E. Hackbarth, Isabel Hardee, Jean M. Johnston, Alanna E. Koehler, Lea Latham, Yvonne L. Latour, C. Christopher Lau, Denise J. Levy, Adam P. Liebendorfer, Ellen F. Macnamara, Valerie V. Maduro, Thomas C. Markello, Alexandra J. McCarty, Jennifer L. Murphy, Michele E. Nehrebecky, Donna Novacic, Barbara N. Pusey, Sarah Sadozai, Katherine E. Schaffer, Prashant Sharma, Sara P. Thomas, Nathanial J. Tolman, Camilo Toro, Zaheer M. Valivullah, Colleen E. Wahl, Mike Warburton, Alec A. Weech, Guoyun Yu, Andrea L. Gropman, David R. Adams, William A. Gahl, May Christine V. Malicdan, Cynthia J. Tifft, Lynne A. Wolfe, Paul R. Lee, John H. Postlethwait, Monte Westerfield, Anna Bican, Rizwan Hamid, John H. Newman, John A. Phillips III, Amy K. Robertson, Joy D. Cogan

\section{Funding}

Research reported in this manuscript was supported by the NIH Common Fund, through the Office of Strategic Coordination/Office of the NIH Director under Award Number U01 HG007709-01 and NIH/NIGMS T32 GM007526 Medical Genetics Research Fellowship Program. This work was funded in part by the US National Human Genome Research Institute (NHGRI)/National Heart Lung and Blood Institute (NHLBI) grant number UM1HG006542 to the Baylor-Hopkins Center for Mendelian Genomics (BHCMG). JEP was supported by NHGRI K08 HG008986 and the Ting Tsung and Wei Fong Chao Foundation. The content is solely the responsibility of the authors and does not necessarily represent the official views of the National Institutes of Health.

\section{Availability of data and materials}

The sequencing data and phenotype datasets analysed during the current study are not publicly available due to privacy concerns, but are available from the corresponding author on reasonable request.

\section{Authors' contributions}

$B L B$ and SRL analysed the data and wrote the manuscript. SRL and BL supervised the study. BLB, JEP, JAR and YY generated and advised on data analysis. BLB, SM, JEP, HES, KG, AB, NPH, KMN, JT, DS, EF, LH, MM, DG, MA MH, SMK, El, SCA, KMJ, JJ, JAR, LE, JRL, JWB, BL and SL identified and collected patients. All authors have read and approved the final manuscript.

\section{Ethics approval and consent to participate}

The institutional review board of the Baylor College of Medicine approved this study. Participants were enrolled after written informed consent was obtained from parents or legal guardians. The study conforms to the Helsinki Declaration.

\section{Consent for publication}

Written informed consent for publication from the parents or legal guardians to publish the work was obtained.

\section{Competing interests}

Baylor College of Medicine (BCM) and Miraca Holdings Inc. have formed a joint venture with shared ownership and governance of the Baylor Genetics (BG), which performs clinical exome sequencing. JEP, MM, JAR, JRL, BL and SRL are employees of BCM and derive support through a professional services agreement with the BG. JRL serves on the Scientific Advisory Board of the BG. JRL has stock ownership in 23andMe, is a paid consultant for Regeneron Pharmaceuticals, has stock options in Lasergen, Inc. and is a co-inventor of US and European patents related to molecular diagnostics for inherited neuropathies, eye diseases and bacterial genomic fingerprinting. The remaining authors declare that they have no competing interests.

\section{Publisher's Note}

Springer Nature remains neutral with regard to jurisdictional claims in published maps and institutional affiliations.

\section{Author details}

'Department of Molecular and Human Genetics, Baylor College of Medicine, 6701 Fannin St, Suite 1560, Houston, TX 77030, USA. ${ }^{2}$ Division of Medical
Genetics, A.I. duPont Hospital for Children/Nemours, Wilmington, DE, USA. ${ }^{3}$ Division of Medical Genetics, Stanford University School of Medicine, Stanford, CA, USA. ${ }^{4}$ Baylor Genetics, Baylor College of Medicine, Houston, TX, USA. ${ }^{5}$ Division of Genetics and Genomic Medicine, Department of Pediatrics, Washington University School of Medicine, St. Louis, MO, USA. ${ }^{6}$ Department of Pediatrics, Section of Medical Genetics, West Virginia University Health Sciences Center, Morgantown, WV, USA. ${ }^{7}$ Children's Hospital of Pittsburgh of UPMC, University of Pittsburgh, Pittsburgh, PA, USA. ${ }^{8}$ Division of Magnetic Resonance Imaging, Silesian Center for Heart Disease, Zabrze, Poland. ${ }^{9}$ The Heart Institute, Cincinnati Children's Hospital Medical Center, Cincinnati, $\mathrm{OH}$, USA. ${ }^{10} \mathrm{NIH}$ Common Fund, Bethesda, MD 20892, USA. ${ }^{11}$ Texas Children's Hospital, Houston, TX 77030, USA. ${ }^{12}$ Department of Pediatrics, Baylor College of Medicine, Houston, TX 77030, USA. ${ }^{13}$ Department of Pediatrics, Baylor College of Medicine, San Antonio, TX 78207, USA. ${ }^{14}$ Human Genome Sequencing Center, Baylor College of Medicine, Houston, TX 77030, USA.

Received: 25 April 2017 Accepted: 24 July 2017

Published online: 14 August 2017

\section{References}

1. Sifrim A, Hitz M, Wilsdon A, Breckpot J, Turki S, Thienpont B, et al. Distinct genetic architectures for syndromic and nonsyndromic congenital heart defects identified by exome sequencing. Nat Genet. 2016;48(9):1060-5.

2. McRae J, et al. Deciphering Developmental Disorders Study. Prevalence and architecture of de novo mutations in developmental disorders. Nature. 2017:542:433-8.

3. Liang K, Gao X, Gilmore JM, Florens L, Washburn MP, Smith E, et al. Characterization of human cyclin-dependent kinase 12 (CDK12) and CDK13 complexes in C-terminal domain phosphorylation, gene transcription, and RNA processing. Mol Cell Biol. 2015;35(6):928-38.

4. Marqués F, Moreau JL, Peaucellier G, Lozano JC, Schatt P, Picard A, et al. A new subfamily of high molecular mass CDC2-related kinases with PITAINRE motifs. Biochem Biophys Res Commun. 2000;279:832-7.

5. Malumbres M, Harlow E, Hunt T, Hunter T, Lahti JM, Manning G, et al. Cyclin-dependent kinases: a family portrait. Nat Cell Biol. 2009;11:1275-6.

6. Blazek D, Kohoutek J, Bartholomeeusen K, Johansen E, Hulinkova P, Luo Z, et al. The Cyclin K/Cdk12 complex maintains genomic stability via regulation of expression of DNA damage response genes. Genes Dev. 2011;25:2158-72.

7. Cao L, Chen F, Yang X, Xu W, Xie J, Yu L. Phylogenetic analysis of CDK and cyclin proteins in premetazoan lineages. BMC Evol Biol. 2014;14:10.

8. Chen H, Lin G, Huang C, Fann M. Cdk12 and Cdk13 regulate axonal elongation through a common signaling pathway that modulates C $d k 5$ expression. Exp Neurol. 2014;261:10-21.

9. White J, Beck C, Harel T, Posey J, Jhangiani S, Tang S, et al. POGZ truncating alleles cause syndromic intellectual disability. Genome Med. 2016;8(1):3.

10. Kohoutek J, Blazek D. Cyclin K goes with Cdk12 and Cdk13. Cell Div. 2012;7(article 12):12.

\section{Submit your next manuscript to BioMed Central and we will help you at every step:}

- We accept pre-submission inquiries

- Our selector tool helps you to find the most relevant journal

- We provide round the clock customer support

- Convenient online submission

- Thorough peer review

- Inclusion in PubMed and all major indexing services

- Maximum visibility for your research

Submit your manuscript at www.biomedcentral.com/submit
Biomed Central 